\title{
Pourbaix-like phase diagram for lithium manganese spinels in acid
}

\author{
R. Benedek, ${ }^{a}$ M. M. Thackeray ${ }^{a}$ and A. van de Walle ${ }^{b}$ \\ Received 16th July 2009, Accepted 8th October 2009 \\ First published as an Advance Article on the web 9th November 2009 \\ DOI: 10.1039/b913226k
}

Calculations are performed on the free energies for proton-promoted reactions of the lithium-ionbattery electrode material $\mathrm{LiMn}_{2} \mathrm{O}_{4}$ spinel in acid, as a function of lithium excess and lithium deficiency relative to stoichiometry. In particular, we consider the dissolution reaction proposed by Hunter (J. Solid State Chem., 1981, 39, 142), in which protons react with lithium manganate spinel to form $\lambda-\mathrm{MnO}_{2}, \mathrm{Li}^{+}$, and $\mathrm{Mn}^{2+}$ products. The calculations employ a hybrid method developed in previous work in which first principles total energy calculations are applied for the solid phases and free atom energies, and tabulated ionization and hydration energies for the aqueous species. A correction to the atomic energies, derived from analysis of binary oxide dissolution reactions, improves the accuracy of the results. A Pourbaix-like dissolution/stability phase diagram is constructed from the resultant reaction free energies.

\section{Introduction}

Protection against acid-promoted dissolution reactions, which corrode lithium-ion-battery cathode materials, ${ }^{1}$ poses a challenge for battery engineering. Although several proposed remedies, such as the application of passivating coatings, ${ }^{2}$ modification of the electrolyte, ${ }^{3}$ and the introduction of selected dopants, ${ }^{4}$ have been found reasonably effective, a better fundamental understanding of the dissolution process may lend additional focus to corrosion protection strategies.

Kinetic (rate) predictions based on phenomenological models of aqueous mineral dissolution ${ }^{5,6}$ are, at best, feasible only with the employment of parameters derived from experiment, which may be unavailable for the materials of interest. In regard to thermodynamics, dissolution-reaction free energies, as a function of $\mathrm{pH}$, are calculable for certain minerals, based on tabulated (compound and aqueous ion) formation free energies. ${ }^{7}$ However, formation free energies for many of the transition metal oxides of interest as potential lithium-ion battery cathodes, particularly at off-stoichiometric compositions, are often unknown. ${ }^{8}$ In this article, we address the thermodynamics of lithium manganese spinel dissolution, using first principles methods.

Based on experimental observation of spinel corrosion in aqueous $\mathrm{HCl}$, Hunter proposed a proton-promoted dissolution reaction ${ }^{9,10}$ for $\mathrm{LiMn}_{2} \mathrm{O}_{4}$,

$$
2 \mathrm{LiMn}_{2} \mathrm{O}_{4}+4 \mathrm{H}^{+} \rightarrow 3 \mathrm{MnO}_{2}+\mathrm{Mn}^{2+}+2 \mathrm{Li}^{+}+2 \mathrm{H}_{2} \mathrm{O}
$$

in which $\mathrm{Li}$ and $\mathrm{Mn}$ ions are leached from the spinel host. Since no divalent Mn occurs in bulk spinel, the reaction must be accompanied by oxidation of the trivalent $\mathrm{Mn}$, with tetravalent $\mathrm{Mn}$ in $\lambda-\mathrm{MnO}_{2}$ as an end-stage product. Reaction (1) neglects the effect of the anions, an idealization, ${ }^{11}$ but a reasonable starting point.

${ }^{a}$ Chemical Sciences and Engineering Division, Argonne National Laboratory, Argonne, IL 60439, USA

${ }^{b}$ Engineering and Applied Science Division, California Institute of Technology, Pasadena, CA, 91125, USA
The thermodynamics of the dissolution reaction (1), and its generalization to off-stoichiometric compositions, is analyzed by the extension of an approach ${ }^{12,13}$ in which solid-phase free energies calculated from first principles are complemented by tabulated aqueous species hydration free energies. ${ }^{14} \mathrm{~A}$ similar treatment is applied to the ion exchange reaction in which protons substitute for lithium on the tetrahedral sites of spinel.

The extension involves a correction to the atomic energies obtained by analysis of binary oxide dissolution. With corrected atomic energies, quantitative accuracy becomes achievable for reaction free energies not heretofore subject to first-principles modeling: despite the widespread success of first principles calculations for chemical reactions and materials properties, there has previously been little success in accounting for reactions that involve ions that change state, in the reaction, between aqueous solution and the solid state, since cancellation of errors cannot be relied upon.

The results are summarized in a phase diagram, which depicts regions of $\mathrm{pH}$-composition space that are either stable or unstable with respect to proton-promoted dissolution. We refer to this diagram as Pourbaix-like, with lithium stoichiometry playing the role played by electrochemical potential in conventional Pourbaix diagrams. ${ }^{15}$ In this work, however, we address only the proton-promoted dissolution of lithium manganese spinels with oxidation states between 3 and 4, which may be viewed as representing a sector of the full Pourbaix diagram.

Sections III, IV and V are concerned with the atomic structure, the acid-promoted dissolution and ion-exchange reactions, and the phase diagram of the spinels. Some further discussion is given in section VI.

\section{Binary-manganese-oxide dissolution}

To test our method, we consider hypothetical dissolution reactions of three binary oxides: trigonally-distorted rocksalt manganosite $\mathrm{MnO}$, spinel $\alpha$-Hausmannite $\mathrm{Mn}_{3} \mathrm{O}_{4}$, and bixbyite $\mathrm{Mn}_{2} \mathrm{O}_{3}$ : 


$$
\mathrm{MnO}+2 \mathrm{H}^{+} \rightarrow \mathrm{Mn}^{2+}+\mathrm{H}_{2} \mathrm{O}
$$

for manganosite,

$$
\mathrm{Mn}_{3} \mathrm{O}_{4}+4 \mathrm{H}^{+} \rightarrow 2 \mathrm{Mn}^{2+}+\mathrm{MnO}_{2}+2 \mathrm{H}_{2} \mathrm{O}
$$

for $\alpha$-Hausmannite, and

$$
\mathrm{Mn}_{2} \mathrm{O}_{3}+2 \mathrm{H}^{+} \rightarrow \mathrm{Mn}^{2+}+\mathrm{MnO}_{2}+\mathrm{H}_{2} \mathrm{O}
$$

for $\beta$-bixbyite.

Although reaction (4), for example, actually proceeds via intermediate $\mathrm{Mn}^{3+}$ ions, ${ }^{16}$ this detail is not relevant to our present purpose, which is to determine the energy balance between reactants and products, for which accurate empirical data ${ }^{7}$ are available to test our first-principles-based approach to reaction free-energy calculation.

\section{A. Empirical reaction free energies}

Employing tabulated formation energies, ${ }^{7}$ we find standard free energies $\Delta G_{\mathrm{r}}^{0}(\mathrm{emp})=-1.06,-1.165,-1.976$, for reactions (2), (3), and (4), in eV per formula unit. We refer to these values as empirical reaction energies, to distinguish them from the hybridmethod calculations described in the following sections. Standard reaction free energies, denoted by the superscript zero, correspond to $\mathrm{pH} 0$, room temperature, and atmospheric pressure. Dissolution reactions (1-4) are all thermodynamically favored (negative free energy change).

\section{B. Hybrid method reaction free energies}

VASP calculations. In the hybrid method, contributions to $\Delta G_{\mathrm{r}}^{0}$ for solid phases ${ }^{12,13}$ are calculated at the GGA $+U$ (generalized gradient approximation corrected for on-site Coulomb interaction $U$ ) level of density-functional theory, as implemented in the VASP (Vienna Ab initio Simulation Package) $\operatorname{code}^{17-19}$ with PAW (projector-augmented wave) pseudopotentials. The PW91 exchange correlation potential ${ }^{20}$ is employed. Convergence with respect to $k$-point sampling was checked for each of the unit-cells employed. The VASP calculations were performed with a basis set cutoff of $500 \mathrm{eV}$.

Our selection of $U_{\text {eff }} \equiv U-J$ in the GGA $+U$ calculations for reactions (2-4) was guided by values obtained from a selfconsistent procedure applied to three lithiated manganese oxide crystal structures relevant to lithium-ion batteries, ${ }^{21}$ and by other literature values. Results would not be markedly different, however, had we used a single $U_{\text {eff }}$ for all binary systems. For convenience, a single value $U_{\text {eff }}=4.84^{21}$ was employed in all calculations for spinels.

The spin configuration of $\mathrm{Mn}$ is treated as ferromagnetic, except for $\mathrm{MnO}$, in which the AFII antiferromagnetic arrangement $\mathrm{t}^{22}$ is employed (with spin direction alternating on successive (111) layers.)

Solid phase free energies. The vibrational free energy, $G_{\text {vib }}(T)$, evaluated at room temperature within the quasi-harmonic approximation, is added to the internal energy at zero temperature, $G_{\text {static }}(T=0)$, to obtain the free energy of a solid phase:

$$
G_{\text {solid }}(T)=G_{\text {static }}(0)+G_{\text {vib }}(T) .
$$

Lattice dynamical analyses of $G_{\mathrm{vib}}(T)$ were performed for several relatively small-unit cell binary and ternary transition metal oxides, and presented in previous work. ${ }^{13,23}$ At room temperature, $G_{\text {vib }}(T)$ was dominated by the zero point energy. The numerical results for these small-unit-cell calculations were consistent with vibrational energies of approximately $0.04 \mathrm{eV}$ per formula unit $\mathrm{Li}, 0.1 \mathrm{eV}$ per formula unit $\mathrm{O}$, and $0.25 \mathrm{eV}$ per formula unit $\mathrm{H}$. By applying these approximations, costly lattice dynamical analyses for conventional-cubic spinel unit cells were avoided, at relatively small loss in accuracy, given other uncertainties in calculated reaction free energies.

Free energies of aqueous species. The aqueous ion free energy, $G\left(\mathrm{aq}_{i}\right)$, is decomposed into three contributions:

$$
G\left(\mathrm{aq}_{i}\right)=E_{\mathrm{ref}}\left(\mathrm{aq}_{i}\right)+E_{\mathrm{ion}}\left(\mathrm{aq}_{i}\right)+G_{\mathrm{hyd}}\left(\mathrm{aq}_{i}\right)
$$

where $E_{\mathrm{ref}}\left(\mathrm{aq}_{i}\right)$ is the atomic (or molecular, in the case of $\mathrm{H}_{2} \mathrm{O}$ ) energy, for the neutral atom ground state, $E_{\mathrm{ion}}\left(\mathrm{aq}_{i}\right)$ is the sum of ionization potentials up to charge state $z\left(\mathrm{aq}_{i}\right)$ and $G_{\mathrm{hyd}}\left(\mathrm{aq}_{i}\right)$ is the hydration free energy ${ }^{14}$ of species aq ${ }_{i}$. (eqn (6) neglects a numerically small free energy contribution associated with the hypothetical expansion of, e.g., $\mathrm{Mn}$ or $\mathrm{Li}$, from densities in the solid to a gaseous density at atmospheric pressure, the reference state pressure in terms of which hydration energies ${ }^{14}$ are defined. ${ }^{24}$ )

Values of $G_{\mathrm{hyd}}\left(\mathrm{aq}_{i}\right)$ taken from experiment are employed for the third term on the RHS of eqn (6). Since the NIST tabulation ${ }^{7}$ of formation free energies, used to evaluate empirical dissolution reaction free energies in section IIA, does not provide explicit hydration energies, a different tabulation ${ }^{14}$ is used. Consequently, a minor inconsistency exists between our reaction free energy calculations with the hybrid-method and those with the empirical thermochemical data, ${ }^{7}$ since the two methods, in effect, are based on (slightly) different hydration energies. The hydration energy for a water molecule is taken from Takahashi $e t a l .^{25}$

Parameters employed in the evaluation of the free energy $G\left(\mathrm{aq}_{i}\right)$ of aqueous species are listed in Table 1.

\section{Correction to hybrid method}

The hybrid-method treatment was found to overestimate the dissolution driving force, $-\Delta G_{\mathrm{d}}^{0}(\mathrm{hyb})$, relative to $-\Delta G_{\mathrm{d}}^{0}(\mathrm{emp})$, in previous analyses of proton-promoted dissolution of $\mathrm{LiCoO}_{2}{ }^{13}$ Analysis of dissolution reactions (2-4) for binary manganese oxides likewise show a higher driving force in hybrid method,

Table 1 Energies (in eV per formula unit) of phases and ionic species that participate in dissolution reactions (2), (4), (7) and (8). $E_{\mathrm{ref}}$ is the neutral atom reference energy, calculated with VASP at the GGA level. $E_{Z}-E_{0}$ is the sum of the first $Z$ ionization energies; ionization energies taken from compilation at the website www.camdb.ac.cn/e/.

\begin{tabular}{llcclc}
\hline species & $E_{\text {ref }}$ & $Z$ & $E_{Z}-E_{0}$ & $E_{\text {hyd }}$ & $E_{Z}+E_{\text {hyd }}$ \\
\hline $\mathrm{H}_{2} \mathrm{O}$ & -14.27 & & & $-0.273[38]$ & -14.54 \\
$\mathrm{H}$ & -1.116 & +1 & 13.6 & $-11.45[14]$ & 1.034 \\
$\mathrm{Mn}$ & -5.059 & +2 & 23.07 & $-19.36[14]$ & -1.35 \\
$\mathrm{Li}$ & -0.270 & +1 & 5.392 & $-5.487[14]$ & -0.365 \\
\hline
\end{tabular}


Table 2 Calculated properties of binary Mn-oxides, based on GGA $+U$ calculations. The hybrid-method dissolution reaction free energy is in third from last column. The corrected values (next to last column), obtained by shifting the Mn reference energy by $1.47 \mathrm{eV}$, are in close agreement with the value derived from empirical data. Lattice constants in $\AA$ free energies in eV

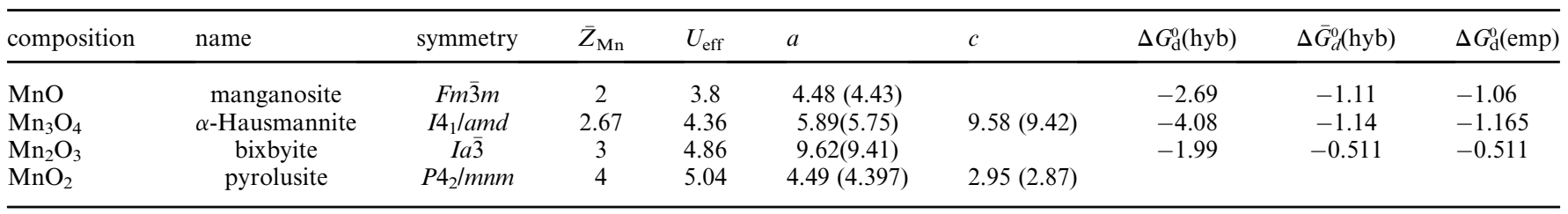

relative to empirical method, calculations, as shown in Table 2 [see columns labeled $\Delta G_{\mathrm{d}}^{0}(\mathrm{hyb})$ and $\Delta G_{\mathrm{d}}^{0}(\mathrm{emp})$ ].

This discrepancy is related to a lack of error cancellation in applications of density functional theory (DFT) to calculate reaction energies when both solid state and atomic (or molecular) species are among the reactants and products. Besides the dissolution reactions considered here, this circumstance also arises in the context of oxidation reactions ${ }^{26}$ and reactive-ion etching. ${ }^{27}$ In effect, the DFT prediction of relative chemical potential of a given chemical species in vapor and solid phases (not calculated explicitly, but implicit in the formulation) contains a significant error, related to the approximate form of the exchange-correlation functional employed. Most existing exchange-correlation functionals were designed for either for atomic and molecular systems or solid state systems, ${ }^{28}$ and are not uniformly accurate for both.

We introduce a correction $\delta E_{\mathrm{ref}}\left(\mathrm{aq}_{i}\right)$ to the atomic reference energy $E_{\mathrm{ref}}\left(\mathrm{aq}_{i}\right)$ to reconcile the hybrid method predictions with empirical reaction free energies, and thereby compensate for the shortcomings of our chosen implementation of DFT. The resultant reference energy is then $\bar{E}_{\mathrm{ref}}\left(\mathrm{aq}_{i}\right)=E_{\mathrm{ref}}\left(\mathrm{aq}_{i}\right)$ $+\delta E_{\mathrm{ref}}\left(\mathrm{aq}_{i}\right)$. Accordingly, we set

$$
\delta E_{\mathrm{ref}}(\mathrm{Mn})=\Delta G_{\mathrm{r}}(\mathrm{emp})-\Delta G_{\mathrm{r}}(\mathrm{hyb})
$$

where the reaction energies are normalized to the number of aqueous $\mathrm{Mn}$ ions on the right hand side of a dissolution reaction. $\delta E_{\text {ref }}$ is intended to correct for the error in the difference between atomic and solid state chemical potentials that results from the adopted approximate form of the exchange correlation functional.

The application of eqn (7) to the binary oxide dissolution reactions, (2-4) yields values of $\delta E_{\text {ref }}(\mathrm{Mn})=1.62,1.46,1.47 \mathrm{eV}$, respectively. The consistency of $\delta E_{\text {ref }}(\mathrm{Mn})$ for the three reactions justifies a posteriori the ad hoc correction procedure based on eqn (7). The value $\delta E_{\mathrm{ref}}\left(\mathrm{aq}_{i}\right)=1.47 \mathrm{eV}$, obtained from reaction (4), is employed (along with the correction $\delta E_{\text {ref }}(\mathrm{Li})=0.35 \mathrm{eV}$ for $\mathrm{Li}^{23}$ in our calculations below for spinel-based systems. Since the corrected reaction free energies, $\Delta \bar{G}_{d}^{0}($ hyb) (next to last column of Table 2), based on $\delta E_{\mathrm{ref}}\left(\mathrm{aq}_{i}\right)=1.47 \mathrm{eV}$, agree closely with empirical values, $\Delta G_{\mathrm{d}}^{0}(\mathrm{emp})$. It is reasonable to expect that the application of shifted atomic reference energies to spinel-based systems would similarly improve accuracy.

\section{Atomic structure of lithium manganese spinel}

Having determined corrected atomic reference energies, $\bar{E}_{\text {ref }}$, we proceed to calculate proton-promoted dissolution and ionexchange reactions of lithium manganese spinel as a function of composition. In this section, we describe the GGA $+U$ calculations for lithium manganese spinels, and some hydrogen substitutions of lithium manganese spinels, which are employed to evaluate solid phase free energies, based on eqn ((5)).

\section{A. Stoichiometric composition}

The crystal structure of stoichiometric $\mathrm{LiMn}_{2} \mathrm{O}_{4}$ is cubic at room temperature, with space group $F d \overline{3} m$. Under the constraint of full cubic symmetry, with $\mathrm{Li}(8 a), \operatorname{Mn}(16 d)$, and $\mathrm{O}(32 e)$, however, $\mathrm{Mn}$ ions assume the unphysical half-integral charge state, $Z_{\mathrm{Mn}}=3.5$. In the real material, dynamical fluctuations break the symmetry, and $\mathrm{Mn}$ disproportionates into trivalent and tetravalent ions. If a small asymmetry is imposed on the initial atomic positions, the GGA $+U$ calculations converge to a disproportionated structure. $^{21,29}$ The oxygen octahedra that enclose trivalent $\mathrm{Mn}$ ions are Jahn-Teller distorted ${ }^{30}$ (see Fig. 1). The resultant calculated GGA $+U$ cell voltages ${ }^{21}$ ( $v s$. Li anode) for a disproportionated arrangement of $\mathrm{Mn}$ are in close agreement with experiment (about $4.15 \mathrm{eV}$ ), whereas the non-disproportionated structure yields cell voltages a few tenths of an $\mathrm{eV}$ less. Lattice constants for $\mathrm{LiMn}_{2} \mathrm{O}_{4}$ predicted by GGA $+U$ are slightly higher than experiment, ${ }^{31-33}$ as shown in Table 3.

\section{B. Lithium substitution for manganese}

Calculations were performed on the family of lithium-manganese spinels, $\mathrm{Li}_{1+x} \mathrm{Mn}_{2-x} \mathrm{O}_{4}$, with lithium substitution on the

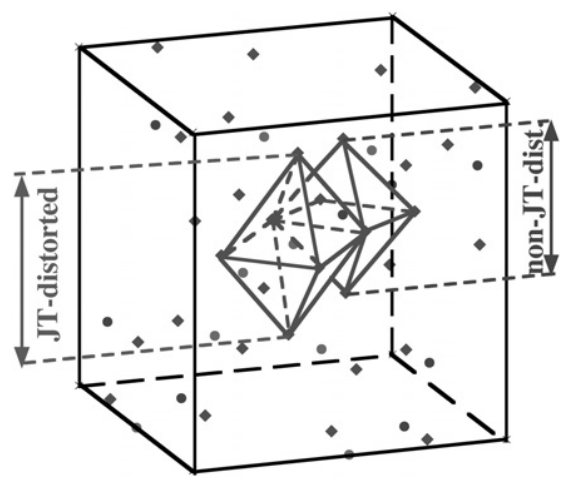

Fig. 1 Atomic structure of conventional cubic unit cell of lithium manganese spinel, $\mathrm{LiMn}_{2} \mathrm{O}_{4}$, calculated with VASP. Li atoms not shown. Mn ions $(\bullet)$ are disproportionated, with half trivalent and half tetravalent. The former are coordinated with Jahn-Teller distorted oxygen ion $(\bullet)$ octahedra. The JT distortion is smaller than in compounds in which $\mathrm{Mn}$ is exclusively trivalent. The arrangement of $3+$ and $4+\mathrm{Mn}$ ions is disordered and not unique. 
Table 3 Calculated properties of over-lithiated spinel $\mathrm{Li}_{1+x} \mathrm{Mn}_{2-x} \mathrm{O}_{4}$ and protonated spinel, $\mathrm{HLi}_{x} \mathrm{Mn}_{2-x} \mathrm{O}_{4}$. Lattice constants in $\stackrel{\circ}{A}$, and reaction energies in $\mathrm{eV}$ per spinel formula unit

\begin{tabular}{lccc}
\hline composition & $a$ & $c$ & $\Delta \bar{G}_{d}^{0}(\mathrm{hyb})$ \\
\hline $\mathrm{LiMn}_{2} \mathrm{O}_{4}$ & $8.415(8.247)[39]$ & & -0.506 \\
$\mathrm{Li}_{9 / 8} \mathrm{Mn}_{15 / 8} \mathrm{O}_{4}$ & 8.373 & -0.427 & 0.237 \\
$\mathrm{Li}_{10 / 8} \mathrm{Mn}_{14 / 8} \mathrm{O}_{4}$ & 8.320 & -0.204 \\
$\mathrm{Li}_{1 / 18} \mathrm{Mn}_{13 / 8} \mathrm{O}_{4}$ & $8.280(8.14)[40]$ & -0.214 \\
$\mathrm{HMn}_{2} \mathrm{O}_{4}$ & 8.476 & & 0.009 \\
$\mathrm{HLi}_{1 / 8} \mathrm{Mn}_{15 / 8} \mathrm{O}_{4}$ & 8.373 & & 0.36 \\
$\mathrm{HLi}_{1 / 4} \mathrm{Mn}_{14 / 8} \mathrm{O}_{4}$ & 8.320 & $9.24(9.274)[31]$ & -2.01 \\
$\mathrm{HLi}_{11 / 8} \mathrm{Mn}_{13 / 8} \mathrm{O}_{4}$ & 8.280 & & -0.299 \\
$\mathrm{Li}_{2} \mathrm{Mn}_{2} \mathrm{O}_{4}$ & $5.77(5.662)[31]$ & & \\
$\mathrm{Li}_{0.5} \mathrm{Mn}_{2} \mathrm{O}_{4}$ & 8.37 & & \\
\hline
\end{tabular}

Mn sublattice. Three such compositions are considered, with $x=$ $1 / 8, x=1 / 4$, and $x=3 / 8$, which correspond to 1,2 , and 3 atomic substitutions in the conventional cubic unit cell, respectively (in real materials, the substitution level is not expected to exceed $x=$ $1 / 3$, which would oxidize the Mn ions beyond the tetravalent state. Substitutional sites on the $16 d$ sublattice were selected to maximize $\mathrm{Li}-\mathrm{Li}$ distances in the cases $x=1 / 4$, and $x=3 / 8$.

\section{Proton substitution for lithium on $8 a$ sublattice}

An alternative reaction to the dissolution of spinel in acid (e.g., reactions (1), or (8), below) is the ion exchange of protons for lithium. For simplicity we have chosen to substitute protons on all $8 a$ sites, rather than consider partially substituted systems. Initial configurations for protonated systems with composition $\mathrm{HLi}_{x} \mathrm{Mn}_{2-x} \mathrm{O}_{4}$ have been simulated by substitution of protons on the $8 a$ sites of the Li-for-Mn substituted materials with composition $\mathrm{Li}_{1+x} \mathrm{Mn}_{2-x} \mathrm{O}_{4}$. Cell parameters and internal coordinates were relaxed to minimize the total energy. The protons form hydroxyl units with bond length about $1 \AA$ in the relaxed structure. ${ }^{34}$ The predicted equilibrium lattice constants for the protonated systems increase relative to the original unsubstituted systems, which suggests that $\mathrm{H}-\mathrm{Li}$ ion exchange is relatively unfavorable energetically. ${ }^{23}$

\section{Over- and under-lithiation on tetrahedral sites}

A tetragonal phase with composition $\mathrm{Li}_{2} \mathrm{Mn}_{2} \mathrm{O}_{4}$ results from adding lithium to $\mathrm{LiMn}_{2} \mathrm{O}_{4}$, which induces a cooperative displacement of Li from tetrahedral to octahedral sites. ${ }^{31}$ An under-lithiated spinel of composition $\mathrm{Li}_{0.5} \mathrm{Mn}_{2} \mathrm{O}_{4}$, which corresponds to an ordered arrangement of the Li vacancies, ${ }^{35}$ was also treated.

\section{Proton-promoted reactions of lithium manganese spinel}

Proton-promoted dissolution ${ }^{12}$ and protonation reactions ${ }^{23}$ of $\mathrm{LiMn}_{2} \mathrm{O}_{4}$ had already been addressed in our earlier work, however, the corrected atomic reference energies introduced in this work greatly improve the accuracy, and enable construction of a realistic dissolution phase diagram for lithium manganese spinels.

\section{A. Li substitution on $16 d$ sublattice}

We consider the proton-promoted dissolution reaction

$$
\begin{aligned}
& 2 \mathrm{Li}_{1+y} \mathrm{Mn}_{2-y} \mathrm{O}_{4}+4(1-y) \mathrm{H}^{+} \rightarrow(3+y) \mathrm{MnO}_{2} \\
& +(1-3 y) \mathrm{Mn}^{2+}+2(1+y) \mathrm{Li}^{+}+2(1-y) \mathrm{H}_{2} \mathrm{O}
\end{aligned}
$$

per formula unit of $\mathrm{Li}_{1+y} \mathrm{Mn}_{2-y} \mathrm{O}_{4}$ and the protonation reaction

$$
\mathrm{LiMn}_{2} \mathrm{O}_{4}+\mathrm{H}^{+} \rightarrow \mathrm{HMn}_{2} \mathrm{O}_{4}+\mathrm{Li}^{+}
$$

\section{B. Other stoichiometries}

Besides the stoichiometric spinel $\mathrm{Li}_{x} \mathrm{Mn}_{2} \mathrm{O}_{4}$, with $x=1$, of special interest are the tetragonal phase ${ }^{36}$ with $x=2$ :

$$
\mathrm{Li}_{2} \mathrm{Mn}_{2} \mathrm{O}_{4}+2 \mathrm{H}^{+} \rightarrow \mathrm{MnO}_{2}+\mathrm{Mn}^{2+}+2 \mathrm{Li}^{+}+2 \mathrm{H}_{2} \mathrm{O}
$$

and the ordered Li-vacancy phase ${ }^{35}$ with $x=0.5$ :

$$
2 \mathrm{Li}_{0.5} \mathrm{Mn}_{2} \mathrm{O}_{4}+4 \mathrm{H}^{+} \rightarrow \frac{7}{2} \mathrm{MnO}_{2}+\frac{1}{2} \mathrm{Mn}^{2+}+\mathrm{Li}^{+}+\mathrm{H}_{2} \mathrm{O}
$$

\section{Phase diagram}

Dissolution free energies, $\Delta \bar{G}_{\mathrm{d}}^{0}(\mathrm{hyb})$, for reactions (8), (10), and (11) and protonation reaction free energies, $\Delta \bar{G}_{p}^{0}(\mathrm{hyb})$, were calculated with the hybrid method, with procedures identical to those applied to binary oxides in section II. Results are listed in Table 3 , in eV per formula unit of the reactant spinel, for reactions (8-11); the tabulated results correspond to $\mathrm{pH} 0$.

At $\mathrm{pH}$ greater than zero, the reaction free energy must be corrected for proton activity: an energy of $2.3 k_{\mathrm{B}} T(\mathrm{pH})$ is added to $\Delta \bar{G}_{d}^{0}(\mathrm{hyb})$, per reactant proton, where $k_{\mathrm{B}}$ is Boltzmann's constant. ${ }^{12}$ In the case of reaction (8), for example, the change in reaction energy at finite $\mathrm{pH}$ is $4.6 \mathrm{pH}(1-y) k_{\mathrm{B}} T$ (per formula unit of reactant spinel). As $\mathrm{pH}$ increases, the reaction free energy becomes less negative, and reverses sign at a critical value, $\mathrm{pH}_{\text {crit }}(y)$ :

$$
\Delta G_{\mathrm{r}}^{0}(y)+4.6 \mathrm{pH}_{\text {crit }}(y)(1-y) k_{\mathrm{B}} T=0
$$

for reaction (8). In the case of the protonation reaction, $\Delta \bar{G}_{p}^{0}(\mathrm{hyb})$ is positive even at $\mathrm{pH} 0$ ( $c f$. Table 3 ), so protonation appears to be not relevant for the equilibrium thermodynamics of spinel. 


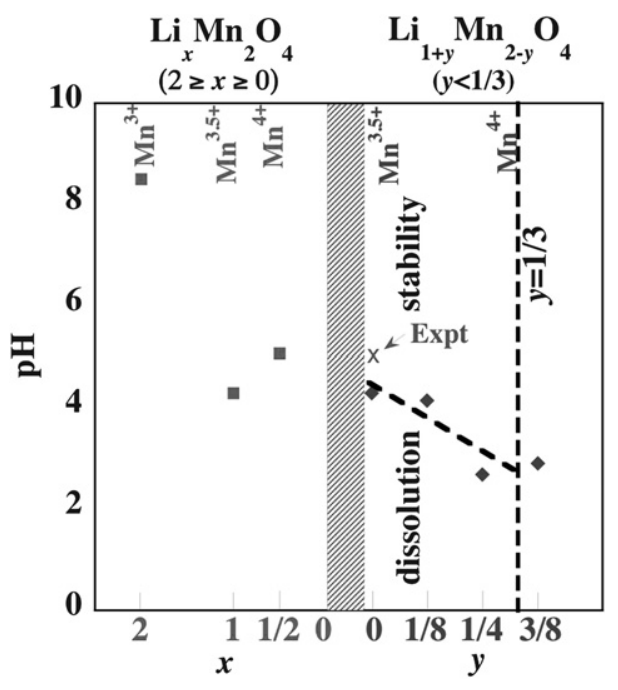

Fig. 2 Phase diagram for lithium manganese spinel in acid, at room temperature, as a function of composition, based on the dissolution reaction free energy calculations described in the text. Left-hand side corresponds to variations of $\mathrm{Li}$ content, with Mn content fixed, and righthand side to Li substitution on Mn sublattice. Calculated points represent $\mathrm{pH}_{\text {crit }}$, below which dissolution occurs, and above which the spinel is stable. The top abscissa scale shows the mean Mn oxidation state at a given composition. The point labeled Expt is from Hunter. ${ }^{9}$ In general stability, against dissolution increases with increasing $\mathrm{Mn}$ oxidation state.

Critical $\mathrm{pH}$ values for dissolution have been calculated for $y=0,1 / 8,1 / 4$, and $3 / 8$, and $x=1 / 2,1$ (equivalent to $y=0$ ) and 2 , by application of eqn (12) and (13) at room temperature. The results are plotted in Fig. 2. The left-hand side of the figure corresponds to the variation of $x$ (non-substitutional $\mathrm{Li}$ ) and the right-hand side, $y$ (Li substitution on Mn sublattice). Dissolution is thermodynamically favored for the regions below the calculated $p H_{\text {crit }}$, whereas regions above it are stable against dissolution. From left to right, the Mn oxidation state increases, and, generally, the region of stability increases correspondingly.

Since $y$ can vary essentially continuously between 0 and $1 / 3$, we draw a dashed line that represents approximately the boundary between dissolution and stability for the lithium-substitution systems. On the other hand, lithium compositions $x=1 / 2$ and 2 correspond to different crystal structures (which represent line compounds), and therefore no interpolated phase boundary line exists between these points.

The point labeled expt in Fig. 2 is taken from the work of Hunter, ${ }^{9}$ who found that dissolution was essentially negligible for $\mathrm{pH}$ above 5 , at stoichiometry $(y=0)$. This appears to be the only experimental data for which a more or less direct comparison with our calculations is possible.

\section{Discussion}

The free energies for proton-promoted dissolution reactions, as well as for proton-for-Li ion-exchange reactions, of spinels of different composition are analyzed in this work. The results of the former calculations may be summarized in a dissolution phase diagram in the composition-pH plane, where the compound composition plays a role analogous to that of electrochemical potential in the Pourbaix diagram of a pure material. Since Pourbaix diagrams ${ }^{15}$ have traditionally been constructed entirely from empirical data, the present approach extends our ability to construct such diagrams to materials where insufficient empirical data are available for the task.

Our method employs solid phase free energies obtained from first principles, and empirically derived hydration energies for aqueous species. A numerical application of this approach to binary-manganese-oxide dissolution showed much improved accuracy when the atomic reference energy $\bar{E}_{\text {ref }}(\mathrm{Mn})$ was adjusted. Adjusted reference energies for $\mathrm{Mn}$ and $\mathrm{Li}$ were therefore employed in calculations for the materials of primary interest in this work, the ternary lithium manganese spinels, and some related proton substituted systems.

The assumed model reactions (1), (8), (10) and (11) neglect anion participation ${ }^{11}$ in the dissolution. Regardless of the precise form of the reaction, one expects anion participation to increase $\mathrm{pH}_{\text {crit }}(x)$ (and therefore extend the region of dissolution in the phase diagram), relative to values calculated for protonpromoted dissolution alone. Since experimental data for the stoichiometric spinel, ${ }^{9} x=1$ is consistent with the calculated $\mathrm{pH}_{\text {crit }}(x=1)$ value plotted in Fig. 2, however, the shift in $\mathrm{pH}_{\text {crit }}(x)$ associated with the anion may not be too pronounced, so that the general trend can be revealed by considering protonpromoted dissolution alone.

Our calculations suggest that proton-Li ion exchange reactions are thermodynamically unfavorable for all spinel compositions considered in this work, for $\mathrm{pH}$ in the acidic range. Electrochemical insertion of protons into spinel has been reported at elevated $\mathrm{pH},{ }^{37}$ and, indeed, proton insertion also occurs in (nonspinel) $\mathrm{MnO}_{2}$ in $\mathrm{Zn}-\mathrm{MnO}_{2}$ alkaline batteries. ${ }^{38}$ The predicted preference of lithium over protons in spinels is consistent with observations that protonated spinels can be employed to extract Li from seawater ${ }^{39}$ by ion exchange.

Finally, we note that the ion-exchange properties of the spinels differ markedly from those of the layered defect-rocksalt phase $\mathrm{Li}_{2} \mathrm{MnO}_{3},{ }^{23,40}$ where the layer stacking adjusts to efficiently bond protons in the layers vacated by $\mathrm{Li}$. We obtain a free energy $\Delta G_{\mathrm{p}}^{0}$ $=-0.35 \mathrm{eV}$ (this value differs slightly from that given in ${ }^{23}$ which did not include $\left.\bar{E}_{\text {ref }}(\mathrm{Mn})\right)$ for the reaction

$$
\mathrm{H}^{+}+\mathrm{Li}_{4 / 3} \mathrm{Mn}_{2 / 3} \mathrm{O}_{2} \rightarrow \mathrm{Li}^{+}+\mathrm{HLi}_{1 / 3} \mathrm{Mn}_{2 / 3} \mathrm{O}_{2}
$$

in contrast to the positive protonation energies listed in Table 3.

\section{Acknowledgements}

The submitted manuscript has been created by UChicago Argonne, LLC, Operator of Argonne National Laboratory ("Argonne"). Argonne, a U.S. Department of Energy Office of Science laboratory, is operated under Contract No. DE-AC0206CH11357. This work was supported at Argonne by the Office of FreedomCar and Vehicle Technologies (Batteries for Advanced Transportation Technologies (BATT) Program), U.S. Department of Energy. Axel van de Walle was supported by the National Science Foundation through TeraGrid computing resources provided by NCSA and SDSC under grant DMR060011N. Grants of computer time at the National Energy 
Research Supercomputer Center, Lawrence Berkeley Laboratory are gratefully acknowledged.

\section{References}

1 W. Choi and A. Manthiram, J. Electrochem. Soc., 2006, 153, A1760.

2 M. M. Thackeray, C. S. Johnson, J. S. Kim, K. C. Lauzze, J. T. Vaughey, N. Dietz, D. Abraham, S. A. Hackney, W. Zeltner and M. A. Anderson, Electrochem. Commun., 2003, 5, 752.

3 W. Li and B. L. Lucht, J. Power Sources, 2007, 168, 258.

4 Y. Liu, X. Li, H. Guo, Z. Wang, Q. Hu, W. Peng and Y. Yang, J. Power Sources, 2009, 189, 721.

5 E. Wieland, B. Wehrli and W. Stumm, Geochim. Cosmochim. Acta, 1988, 52, 1969.

6 M. A. Blesa, P. J. Morando, and A. E. Regazzoni, Chemical Dissolution of Metal Oxides(CRC Press, Boca Raton, FL, 1993).

7 D. D. Wagman, W. H. Evans, V. B. Parker, R. H. Schumm, I. Halow, S. M. Bailey, K. L. Churney and R. L. Nuttall, J. Phys. Chem. Ref. Data, 1982, 11, 2.

8 L. A. Reznitskii, Russian Journal of Inorganic Chemistry, 2003, 48, 233.

9 J. C. Hunter, J. Solid State Chem., 1981, 39, 142.

10 E. Wang, D. Ofer, W. Bowden, N. Iltchev, R. Moses and K. Brandt, J. Electrochem. Soc., 2000, 147, 4023.

11 A. Fedorockova and P. Raschman, Chem. Eng. J., 2008, 143, 265.

12 R. Benedek and M. M. Thackeray, Electrochem. Solid-State Lett., 2006, 9, A265.

13 R. Benedek and A. van de Walle, J. Electrochem. Soc., 2008, 155, A711.

14 W. R. Fawcett, J. Phys. Chem. B, 1999, 103, 11181.

15 G. Stansbury and R. Buchanan, Fundamentals of Electrochemical Corrosion(ASM International, Materials Park, OH, 2000).

16 D. K. Walanda, G. A. Lawrance and S. W. Donne, J. Solid State Chem., 2009, 182, 1336.

17 G. Kresse and J. Furthmueller, Comput. Mater. Sci., 1996, 6, 15.

18 G. Kresse and J. Furthmueller, Phys. Rev. B: Condens. Matter, 1996, 54, 11169 .

19 G. Kresse and D. Joubert, Phys. Rev. B: Condens. Matter Mater. Phys., 1999, 59, 1758.
20 Y. Wang and J. P. Perdew, Phys. Rev. B: Condens. Matter, 1991, 44, 13298.

21 F. Zhou, C. Cococcioni, C. A. Marianetti, G. Morgan and G. Ceder, Phys. Rev. B: Condens. Matter Mater. Phys., 2004, 70, 235121.

22 J. E. Pask, D. J. Singh, I. I. Mazin, C. S. Hellberg and J. Kortus, Phys. Rev. B: Condens. Matter Mater. Phys., 2001, 64, 024403.

23 R. Benedek, M. M. Thackeray and A. van de Walle, Chem. Mater., $2008,20,5485$.

24 Y. Marcus and J. Chem, J. Chem. Soc., Faraday Trans., 1991, 87, 2995.

25 H. Takahashi, N. Matubayasi, M. Nakahara and T. Nitta, J. Chem. Phys., 2004, 121, 3989.

26 L. Wang, T. Maxisch and G. Ceder, Chem. Mater., 2007, 19, 543.

27 N. Ozawa, T. Roman, M. David, H. Kishi and H. Kasai, J. Phys.: Condens. Matter, 2008, 20, 355006.

28 J. Hafner, J. Comput. Chem., 2008, 29, 2044.

29 C. Y. Ouyang, S. Q. Shi and M. S. Lei, J. Alloys Compd., 2009, 474, 370.

30 A. Marianetti, D. Morgan and G. Ceder, Phys. Rev. B: Condens. Matter Mater. Phys., 2001, 63, 224304.

31 J. B. Goodenough, M. M. Thackeray, W. I. F. David and P. G. Bruce, Rev. de Chimie minerale, 1984, t. 21, 435.

32 B. Ammundsen, G. R. Burns, M. S. Islam, H. Kanoh and J. Roziere, J. Phys. Chem. B, 1999, 103, 5175.

33 B. Ammundsen, D. J. Jones, J. Roziere and G. R. Burns, Chem. Mater., 1995, 7, 2151.

34 C. M. Fang and G. A. de Wijs, Chem. Mater., 2006, 18, 1169.

35 C. Y. Ouyang, S. Q. Shi, Z. X. Wang, H. Li, X. J. Huang and L. Q. Chen, Europhys. Lett., 2004, 67, 28.

36 W. I. F. David, J. B. Goodenough, M. M. Thackeray and M. G. S. R. Thomas, Rev. de Chimie minerale, 1983, t. 20, 636.

37 C. Cachet-Vivier, S. Bach and J. P. P. Pereira-Ramos, Electrochim. Acta, 1999, 44, 2705.

38 M. Minakshi, P. Singh, M. Carter and K. Prince, Electrochem. SolidState Lett., 2008, 11, A145.

39 H. Koyanaka, O. Matsubaya, Y. Koyanaka and N. Hatta, J. Electroanal. Chem., 2003, 559, 77.

40 Y. Paik, C. P. Grey, C. S. Johnson, J. S. Kim and M. M. Thackeray, Chem. Mater., 2002, 14, 5109. 